\title{
Collaborative Signal Multiple Access: A Robust Alternative to NOMA Using SIC
}

\author{
Indu L. Shakya, and Falah H. Ali, Senior Member, IEEE
}

\begin{abstract}
We propose a novel non-orthogonal multiple access scheme referred to as collaborative signal multiple access (CSMA) to address key challenges of non-orthogonal multiple access (NOMA) enabling its use for future 5G/6G mobile systems. It provides higher capacity and robust operation in diverse wireless systems. A base-station groups small number of users to transmit their data simultaneously using power and signal space domains. A receiver performs collaborative joint detection of all users' data using minimum Euclidean distance criterion to extract only the desired user's data with minimal loss of signal energy. This approach is able to tolerate much wider power differences to operate in extreme co-channel interference scenarios where NOMA using successive interference cancellation (SIC) fails even under infinite signal-to-noise ratio (SNR). We provide extensive analyses and simulations supported by upper bounds on symbol error rates (SER) for different power allocation and M-QAM constellations. Numerical results confirm superiority of the proposed scheme in error performance and resulting capacity compared to NOMA, making it a very robust and practical alternative.
\end{abstract}

Index Terms-NOMA, high capacity, successive interference cancellation, M-QAM, joint detection

\section{INTRODUCTION}

$\mathbf{N}$ OMA has received widespread attention as being a promising approach to increase the sum rates of wireless access [1]- [7]. Interests on practical implementation of NOMA are also growing as evidenced by its inclusion as a study-item in 3GPP [2], [3]. It relies on the SIC method to cancel inter-user interference and is known to outperform orthogonal multiple access schemes in terms of sum rates when channel gain disparity between users are high [1]. However for the SIC method to work reliably it needs to meet very stringent conditions of power distribution among users, or else, it fails to decode its own user data due to high interference [5], not to mention the fact that grouping of users becomes very challenging [7], [10]. Furthermore, near equal power allocation and non-degraded broadcast channel condition represent other acute problems for NOMA SIC receivers as they are unable to decode the interference and hence ends up with error floor [6]. These issues are only excerbated as the number of users

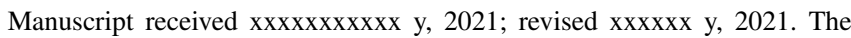

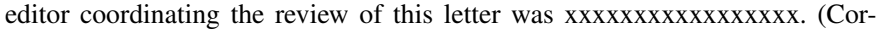
responding author: Indu L. Shakya.)

Indu L. Shakya was with the University of Sussex, Brighton, BN1 9QT UK. He is now a technology leader and consultant for wireless and satellite communications industries (ishakya@gmail.com). He is also a research collaborator with the Communications Research Group, University of Sussex, Brighton, BN1 9QT, UK. Falah H. Ali is a Professor of Communications Engineering, at the School of Engineering and Informatics, University of Sussex, Brighton, BN1 9QT UK. (f.h.ali@sussex.ac.uk). grow. Therefore NOMA is still not included as work-item in the 3GPP and left possible inclusion in beyong 5G [4].

We address some of these critical issues of NOMA with a new collaborative approach called CSMA-where users' signals are not treated as interference or noise-and detail its system design. This is inspired by the authors' early work on CDMA multi-user detection problem [9]. The CSMA approach makes use of the knowledge of all users' transmitted modulation symbol alphabets and power information to jointly detect and estimate all users' data by exploiting the fact that any of the possible location of a composite received signal is uniquely identifiable on the complex plane. A distinct difference between CSMA and NOMA SIC is, the latter requires received constellation points between each nearest neighbours to be within fixed partitions to decode user data and regenerate interference. However with CSMA, any of the constellation points can be unevenly spaced to support much wider range of power allocated to users at the transmitter. Unlike most of work on the NOMA literature e.g. [1], [5], [6] which require strict user power control to ensure SIC method works satisfactorily, we show here that CSMA can operate well even under the equal power for users and maintain decent SER. We provide theoretical analysis of SER of CSMA using minimum distance method to derive SER upper bounds and validate them with numerical simulations. Furthermore, we provide extensive comparative studies to confirm its superiority against NOMA in large majority of practical cases.

\section{Signal AND System Model}

We consider a downlink system with $K$ users being served by a single base-station (BS). The composite conetellation of $K$ users $s$, transmitted from the BS as shown in Figure 1, can be written as:

$$
s=\sum_{k=1}^{K} \sqrt{\alpha_{k} P_{t}} x_{k},
$$

where $\alpha_{k}$ is a power allocation coefficient consisting of a fraction of the total power $P_{t}$ such that $\sum_{k=1}^{K} \alpha_{k}=1$. The constituent modulated symbol of each user $x_{k}$ is taken from a square M-QAM constellation with symmetric in-phase $i_{k}$ and quadrature $q_{k}$ components as follows:

$$
x_{k}=i_{k}+\sqrt{-1} q_{k} .
$$

Each user's symbols have alphabet of size $M_{k}$ i.e. $x_{k} \in \mathbb{X}_{k}=\left[x_{1}, x_{2}, . . x_{M_{k}}\right]$ where the in-phase and quadrature components, each are made of independent pulse amplitude 


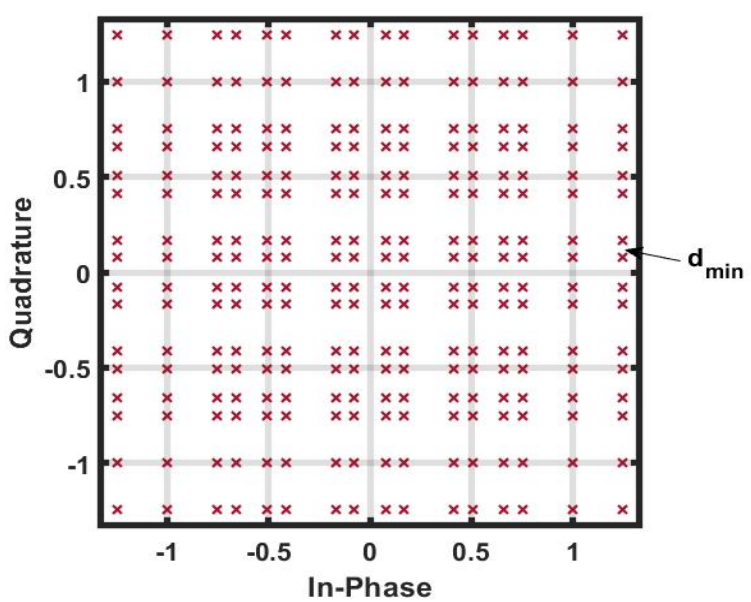

Fig. 1. Composite signal constellation diagram for 2-user CSMA with 16QAM constituent modulations $\left(M_{1}=M_{2}=16\right)$ and unequal power allocation of $\alpha_{1}=0.85$ and $\alpha_{2}=0.15$. Normalized minimum distance of the composite signal $d_{\min }=0.0932$.

modulated signals that are evenly spaced for square constellations as follows:

$$
\begin{aligned}
& i_{k}\left(n_{k}\right)=\left(2 n_{k}-1\right) C_{k}, n_{k}=-\frac{\sqrt{M_{k}}}{2}+1, \ldots, \frac{\sqrt{M_{k}}}{2}, \\
& q_{k}\left(n_{k}\right)=\left(2 n_{k}-1\right) C_{k}, n_{k}=-\frac{\sqrt{M_{k}}}{2}+1, \ldots, \frac{\sqrt{M_{k}}}{2} .
\end{aligned}
$$

The modulated symbols are then multiplied by the modulation specific scaling factor $C_{k}$ and user specific power allocation $\alpha_{k}$ to ensure unity expected energy $\mathbb{E}\left\{|x|^{2}\right\}=1$ of composite signal. This will ensure that the CSMA composite signals maintain the same average and peak power constraints as in single-user case. The BS shares $\alpha_{k}, M_{k}$ over control channels. The $C_{k}$ value is calculated as follows:

$$
C_{k}=\sqrt{\frac{3 \alpha_{k} P_{t}}{2\left(M_{k}-1\right)}}
$$

The signal at the receiver of user $k$ can be written as:

$$
r_{k}=g_{k} s+z_{k},
$$

where $g_{k}$ represents baseband model of complex fading channel between the BS and user $k$. With this model we can safely remove pathloss parameters to focus study on interference mitigation aspects while still accounting for short-term fading effects. We assume independent Rayleigh distribution for fading channels with $\mathcal{C N}(0,1)$. Finally, $z_{k}$ represents the additive white Gaussian noise (AWGN) component with $\mathcal{C N}\left(0, N_{0}\right)$.

\section{Proposed CSMA Receiver DeSign}

In the following, we first highlight the features of NOMA SIC method first to compare and contrast with our collaborative receiver.

\section{A. Existing NOMA SIC Receivers}

The SIC method works on the principle that the users that are closer to the BS achieve higher SNR compared with users that are further away. Hence a receiver for user $l$ can readily decode the data sent to far users $l+1, . ., K$, then use the estimated data $\hat{x}_{l+1}, \ldots, \hat{x}_{K}$ to regenerate their interference and subtract the sum from the total received signal $r_{l}$ in the SIC process, to form a more reliable estimate $\hat{x}_{l}$ as follows:

$$
\hat{x}_{l}=\arg \min _{x_{l} \in \mathbb{X}_{l}}\left|r_{l}-\sum_{k=l+1}^{K} \hat{h}_{l} \sqrt{\alpha_{k} P_{t}} \hat{x}_{k}-\hat{h}_{l} \sqrt{\alpha_{l} P_{t}} x_{l}\right|^{2} .
$$

Usually the near users are allocated lower power e.g. $\alpha_{1}<$ $\alpha_{K}$. Therefore the user $K$ that is located furthest from the $\mathrm{BS}$, is allocated the highest fraction of the total power $\alpha_{K}$. This receiver simply decodes the user's data by treating other users' data as AWGN, which reduces to performing singleuser detection over $M_{K}$ possibilities as follows:

$$
\hat{x}_{K}=\arg \min _{x_{K} \in \mathbb{X}_{K}}\left|r_{K}-\hat{h}_{K} \sqrt{\alpha_{K} P_{t}} x_{K}\right|^{2} .
$$

For the SIC to work satisfactorily, a strict power alocation condition of users must be met [5], [6]. For the case of $K=2$, assuming user 2 has worse channel than user 1 , the power allocation rule is given in [6] as follows:

$$
\alpha_{2}>\frac{\left(M_{1}-1\right)\left(\sqrt{M_{2}}-1\right)^{2}}{M_{2}-1+\left(M_{1}-1\right)\left(\sqrt{M_{2}}-1\right)^{2}},
$$

while $\alpha_{1}=1-\alpha_{2}$.

\section{B. Proposed CSMA Receiver}

In the proposed CSMA approach, each receiver extracts data of the desired user $\hat{x}_{k}$ as well as other users $x_{i}, i=$ $1,2, . . K, i \neq k$ jointly from $r_{k}$ by comparing $r_{k}$ against composite signal from all possible transmitted data combinations $\mathbb{X}_{i}, i=1,2, . ., K$ to identify the desired data $\hat{x}_{k}$ as follows:

$$
\hat{x}_{k}=\arg \min _{x_{1} \in \mathbb{X}_{1}, \ldots x_{K} \in \mathbb{X}_{K}}\left|r_{k}-\hat{h}_{k} \sum_{i=1}^{K} \sqrt{\alpha_{i} P_{t}} x_{i}\right|^{2} .
$$

With the above detection method, the receiver is able to separate users' data as long as their possible composite signals are not identical. Note that the proposed receiver requires $\prod_{k=1}^{K} M_{k}$ computations per symbol period which is normally higher than SIC. However for the practical SNR ranges and $K$ and $M_{k}$ normally being small, this is still affordable. See Table I for an example for $K=3$ and $\left\{M_{1}, M_{2}, M_{3}\right\}=\{4,4,4\}$.

\section{Minimum Distance And SER Analyses}

It is known from [11] that SER of single user M-QAM serves as lower bound on SERs for any multiuser systems, which also apples to CSMA. For deriving the upper bound, we make use of a normalised squared minimum distance $\bar{d}_{C}^{2}$ as a metric to quantify its effect on SER of the proposed receiver so we can plug it into our analysis later. An example of $d_{m i n}$ among all nearest neighbors is shown in Figure 1 for $K=2$ and $M_{1}=M_{2}=16, \alpha_{1}=0.85, \alpha_{2}=0.15$. The $\bar{d}_{C}^{2}$ is 
obtained by selecting the minimum of all calculated distances as follows:

$$
\bar{d}_{C}^{2}=\min \left\{d_{a, b}^{2}\right\}, \forall a, \forall b, a \neq b,
$$

where $d_{a, b}^{2}$ are the calculated distances against an array of all possible $K$ user data combinations of size $\prod_{k}^{K} M_{k}$ against all other $\prod_{k}^{M} M_{k}-1$ possibilities.

$$
\begin{array}{r}
d_{a, b}^{2}=\left|\sum_{k=1}^{K} \alpha_{k} P_{t} x_{k}^{a}-\sum_{k=1}^{K} \alpha_{k} P_{t} x_{k}^{b}\right|^{2}, a \neq b, \\
x_{k}^{a} \in \mathbb{X}=\left\{\left[x_{1}^{1}, x_{2}^{1}, \ldots x_{K}^{1}\right]\left[x_{1}^{2}, x_{1}^{1}, \ldots x_{K}^{1}\right] \ldots\left[x_{K}^{M_{1}}, x_{2}^{M_{2}}, \ldots x_{K}^{M_{K}}\right]\right\} ; \\
x_{k}^{b} \in \mathbb{X}=\left\{\left[x_{1}^{1}, x_{2}^{1}, \ldots x_{K}^{1}\right]\left[x_{1}^{2}, x_{1}^{1}, \ldots x_{K}^{1}\right] \ldots\left[x_{K}^{M_{1}}, x_{2}^{M_{2}}, \ldots x_{K}^{M_{K}}\right]\right\} .
\end{array}
$$

To compare and build upon the single-user case, we obtain comparable minimum distance value for a square M-QAM modulated symbols [11] of alphabet size $M_{\Sigma}=\prod_{k=1}^{K} M_{k}$ that can be used as a reference super-constellation, as follows:

$$
\bar{d}_{M_{\Sigma}}^{2}=\frac{6}{M_{\Sigma}-1} .
$$

Therefore the SER of CSMA can be obtained by utilizing the $\bar{d}_{C}^{2}$ in relation to $\bar{d}_{M_{\Sigma}}^{2}$ to produce a a weighted $E_{b} / N_{0}$ for CSMA $\gamma_{C}^{b}$, as follows:

$$
\gamma_{C}^{b}=\frac{\bar{d}_{C}^{2}}{\bar{d}_{M_{\Sigma}}^{2}} \frac{\gamma}{\log _{2} M_{\Sigma}},
$$

where $\gamma=\frac{P_{t}}{N_{0}}$ is the total SNR. The $\gamma_{C}^{b}$ derived here can be seen as having an effect of shifting the SNR axis for CSMA by $\frac{\bar{d}_{C}^{2}}{\bar{d}_{M_{\Sigma}}^{2}}$ while keeping the same SER trend of a single user detection as long as $\bar{d}_{C}^{2}>0$. A probability of making a symbol eror for CSMA can be then obtained after doing some manipulations on the expressions given [8] while using $\gamma_{C}^{b}$ instead of $\gamma$ as follows:

$$
\begin{array}{r}
P e_{C}=\frac{\sqrt{M_{\Sigma}}-1}{M_{\Sigma}}\left\{\left(\sqrt{M_{\Sigma}}-1\right)+4\left(\begin{array}{c}
1-\mu_{C} \\
2
\end{array}\right)\right. \\
\left.-\left(\sqrt{M_{\Sigma}}-1\right)\left\{\frac{4}{\pi} \mu_{C} \tan ^{-1}\left(\mu_{C}\right)\right\}\right\},
\end{array}
$$

where $\mu_{C}=\sqrt{\frac{\beta}{1+\beta \gamma_{C}^{b}}}$ with $\beta=\frac{3 \log 2\left(M_{\Sigma}\right)}{2\left(M_{\Sigma}-1\right)}$. Since $P e_{C}$ in (15) uses the minimum distance measure, it is expected that SER obtained from numerical simulations will be upper bounded by $\mathrm{Pe}_{C}$; tightness of which depends of the distribution of distance values.

\section{NUMERICAL RESUlTS AND DISCUSSIONS}

For further analysis and comparisons with NOMA, we use simulations assuming the following setup. We use two-user system for clarity and ease of comparsons, $M_{k} \in\{4,16\}$ and vary $\alpha_{1} \in[0.05-0.5]$ where $\alpha_{2}=1-\alpha_{1}$. Channel estimation is assumed perfect $\hat{g}_{k}=g_{k}, \forall k$.
TABLE I

EXAMPle Of COMPUTATIONAL EFForTS Of CSMA Against NOMA

\begin{tabular}{c|c|c}
\hline Scheme & Detection and Demodulation & Operations \\
\hline \hline NOMA SIC [1] & $2 M_{3}+2 M_{2}+M_{1}$ & 20 \\
\hline CSMA & $M_{1} M_{2} M_{3}$ & 64 \\
\hline
\end{tabular}

TABLE II

NORMALIZED SQUARED MINIMUM DISTANCES $\bar{d}_{C}^{2}$, OF CSMA FOR $K=2$ AND DIFFERENT $M_{k}$ AND $\alpha_{k}$ VALUES. AS REFERENCE, SINGLE USER SQUARE MQAM SYMBOLS WITH $M_{\Sigma}=4,16,64,256$ GIVE $\bar{d}_{\Sigma}^{2}$ OF $2,0.4,0.0952,0.0235$, RESPECTIVELY

\begin{tabular}{c|c|c|c|c|c}
\hline$M_{1}, M_{2}$ & $\alpha_{1}, \alpha_{2}$ & $\alpha_{1}, \alpha_{2}$ & $\alpha_{1}, \alpha_{2}$ & $\alpha_{1}, \alpha_{2}$ & $\alpha_{1}, \alpha_{2}$ \\
\hline$a) 4,4$ & $0.15,0.85$ & $0.2,0.8$ & $0.3,0.7$ & $0.4,0.6$ & $0.5,0.5$ \\
\hline $\bar{d}_{C}^{2}$ & 0.3 & 0.4 & 0.17 & 0.04 & 0 \\
\hline \hline$b) 4,16$ & $0.15,0.85$ & $0.2,0.8$ & $0.3,0.7$ & $0.4,0.6$ & $0.5,0.5$ \\
\hline $\bar{d}_{C}^{2}$ & 0.0013 & 0.0045 & 0.0062 & 0.0073 & 0.011 \\
\hline \hline$c) 16,4$ & $0.15,0.85$ & $0.2,0.8$ & $0.3,0.7$ & $0.4,0.6$ & $0.5,0.5$ \\
\hline $\bar{d}_{C}^{2}$ & 0.06 & 0.08 & 0.02 & 0.010 & 0.011 \\
\hline \hline$d) 16,16$ & $0.15,0.85$ & $0.2,0.8$ & $0.3,0.7$ & $0.4,0.6$ & $0.5,0.5$ \\
\hline $\bar{d}_{C}^{2}$ & 0.0087 & 0 & 0.0004 & 0.0235 & 0 \\
\hline
\end{tabular}

In Table II, we populate $\bar{d}_{C}^{2}$ values for difrerent $\alpha_{1}, \alpha_{2}$ for the combinations a) $M_{1}=4, M_{2}=4$, b) $M_{1}=4, M_{2}=$ 16, c) $M_{1}=16, M_{2}=4$ and d) $M_{1}=16, M_{2}=16$. For the case of a) $M_{1}=4, M_{2}=4$, the best $\bar{d}_{C}^{2}$ is obtained for $\alpha_{1}=0.2, \alpha_{2}=0.8$ which is also the $\bar{d}_{M_{\Sigma}}^{2}$ of 16-QAM. The $\bar{d}_{C}^{2}$ reduced to 0 when $\alpha_{1}=0.5, \alpha_{2}=0.5$ as some of the composite signals become identical, resulting in detection ambiguity. The $\bar{d}_{C}^{2}$ values for b) $M_{1}=4, M_{2}=16$, are worse than that of c) $M_{1}=16, M_{2}=4$ for all cases of $\alpha_{1}, \alpha_{2}$, except for $\alpha_{1}=0.5, \alpha_{2}=0.5$. This is because, a subset of neighbouring signal points become spaced much closer to each other in the former than in the latter case. Conversely, the combination c) $M_{1}=16, M_{2}=4$ can achieve up to $\bar{d}_{C}^{2}=0.08$ which is very close to $\bar{d}_{M_{\Sigma}}^{2}=0.0952$ of 64-QAM. Finally, $\bar{d}_{C}^{2}$ for d) $M_{1}=16, M_{2}=16$ are given, where the best $\bar{d}_{C}^{2}$ is obtained for $\alpha_{1}=0.4, \alpha_{2}=0.6$.

Figure 2 shows the SER of CSMA for $K=2$ and $M_{1}=16, M_{2}=4$, with two users labeled as $\mathrm{U} 1$ and $\mathrm{U} 2$, respectively for different $\alpha_{1}, \alpha_{2}$ values and how they compare against NOMA under the same total SNR, $P_{t} / N_{0}$. The SER upper bounds calculated using (15) are also plotted. The SER curves for U1 with $\alpha_{1}=0.17$ and U2 with $\alpha_{2}=0.83$ are the same for both NOMA and CSMA. This is because with the $\alpha_{2}=0.83$, the condition in (9) is satisfied and SIC can detect and cancel all interference fully. This indicates CSMA and NOMA achieve identical SERs when composite signals are fully separated. The SER of U2-which is allocated more power-is better than U1 as expected. The CSMA SER upper bounds obtained from (15) are plotted to show how tight these upper bounds are. The $P e_{C}$ curve for $\alpha_{1} / \alpha_{2}=0.17 / 0.83$ matches the simulated SER very well, indicating that distances are evenly distributed around $\bar{d}_{C}^{2}$. The $P e_{C}$ for $\alpha_{1} / \alpha_{2}=0.3 / 0.7$ are higher than that of simulated SERs. This can be attributed to the uneven distibution of distances with more points giving distances greater than $\bar{d}_{C}^{2}$. It is worth noting that the SER performance of CSMA depends only on how closely spaced all the possible composite signals 


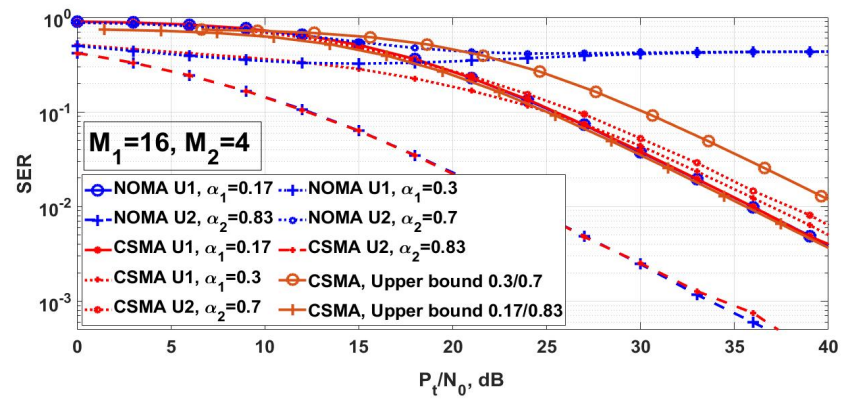

Fig. 2. SER of users U1 and U2 for CSMA compared with NOMA for $K=2$, for $M_{1}=16, M_{2}=4$ and different $\alpha_{1}, \alpha_{2}$ values.

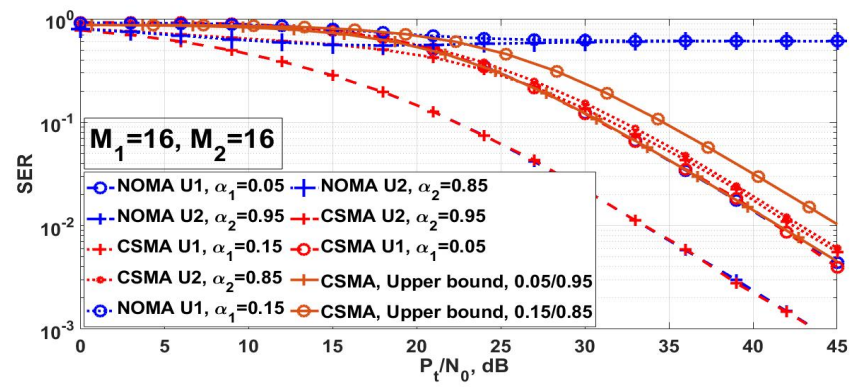

Fig. 3. SER of users $\mathrm{U} 1$ and $\mathrm{U} 2$ for CSMA compared with NOMA for $K=2$, for $M_{1}=16, M_{2}=16$ and different $\alpha_{1}, \alpha_{2}$ values.

of users' are relative to each other and the magnitude of fading channels as there is no interference term as in NOMA. Therefore CSMA can operate without exhibiting error floor for $\alpha_{1}=0.3$ and $\alpha_{2}=7$ as it can identify users' data even if the composite signals cross the boundaries of fixed partitions unlike NOMA SIC.

Figure 3 shows the SER of CSMA for $K=2$ and $M_{1}=16, M_{2}=16$, with two users labeled as U1 and U2, respectively for different $\alpha_{1}, \alpha_{2}$ values and how they compare against NOMA under the same total SNR, $P_{t} / N_{0}$. The SER upper bounds calculated using (15) are also plotted. Similar conclusions to that in Figure 2 can be made here. The bound $P e_{C}$ for $\alpha_{1} / \alpha_{2}=0.15 / 0.85$ show higher SER than simulated SER but the gap is less. This can be attributed to higher concentration of $d_{a, b}^{2}$ that are closer to $\bar{d}_{C}^{2}$ than in the previous case.

In Figure 4, we plot the simulated SERs of CSMA for a) $M_{1}=16, M_{2}=4$, b) $M_{1}=16, M_{2}=16$, for $\alpha_{1}$ values between $[0.05-0.5]$ to compare its robustness against NOMA under the same conditions. It is clear that CSMA offers much better SER than NOMA, specially when $\alpha_{1} \rightarrow \alpha_{2}$. This is because, as more composite signals from the constellation points cross the fixed boundaries NOMA cannot distinguish the desired and other user data and hence fails to show satisfactory SERs. CSMA on the contrary can separate the users' data well even when they are allocated equal powers i.e. $\alpha_{1}=\alpha_{2}=0.5$ for $M_{1}=16, M_{2}=4$ and almost equal e.g. $\alpha_{1}=0.4, \alpha_{2}=0.6$ for the case of $M_{1}=16, M_{2}=16$ as $\bar{d}_{C}^{2}>0$ in both cases. CSMA performance degrades for a few $\alpha_{1}$ values e.g. $0.1,0.2,0.35$ as $\bar{d}_{C}^{2} \rightarrow 0$. In such cases, SER can be improved by rotating phase of users' symbols. For example, for $M_{1}=M_{2}=16$ and $\alpha_{1}=0.2, \alpha_{2}=0.8$

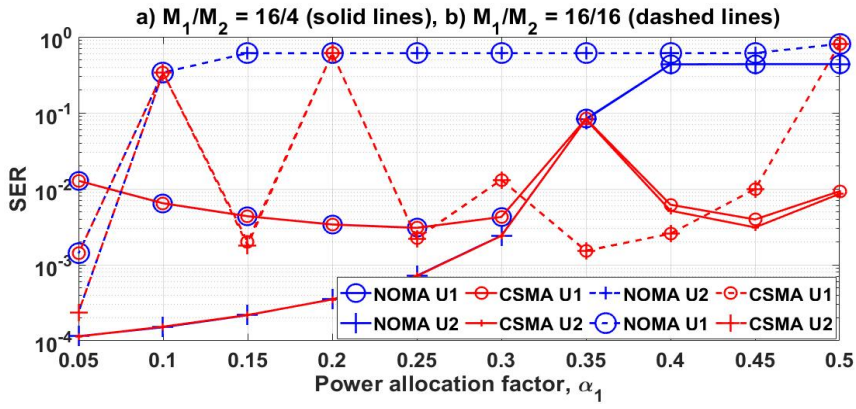

Fig. 4. SER performances of CSMA for users U1 and U2 compared with that of NOMA for a) $M_{1}=16, M_{2}=4, P_{t} / N_{0}=40 \mathrm{~dB}$ (solid lines), and b) $M_{1}=16, M_{2}=16, P_{t} / N_{0}=50 \mathrm{~dB}$ (dashed lines), for different $\alpha_{1}$ within the range $[0.05-0.5]$ where $\alpha_{2}=1-\alpha_{1}$.

as in Table II, an improved $\bar{d}_{C}^{2}=0.0123$ can be achieved by shifting phase of user 1 by $\pi / 16$ degrees.

\section{CONCLUSIONS}

We proposed a novel CSMA approach that is much more robust than NOMA in practical wireless environments and hence suitable for diverse applications. It is shown to be able separate users' data even under extreme interference conditions to relax the requirement on grouping of users and depent less on their propagation channel disparity that is a must for NOMA. In terms of throughput, it can simultaenously support e.g. two users over much wider power ranges to give sum rate of 8 $\mathrm{bps} / \mathrm{Hz}$ at a SER of $1 e-2$ where the NOMA operation range is very small. We have demonstrated the proof of concept of the proposed technique utilizing single antennas and standard M-QAM constellations. However, system performance could be easily improved by integrating phase rotation and standard MIMO techniques. This is the subject for future work.

\section{REFERENCES}

[1] Y. Saito et al., "Non-Orthogonal Multiple Access (NOMA) for Future Radio Access," Proc. IEEE VTCSpring '13, June 2013, pp. 1-5.

[2] 3rd Generation Partnership Project (3GPP), "Study on downlink multiuser superposition transmission for LTE," Mar. 2015.

[3] 3rd Generation Partnership Project (3GPP), "Study on non-orthogonal multiple access (NOMA) for NR (Release 16)," Dec. 2018.

[4] B. Makki, K. Chitti, A. Behravan and M. -S. Alouini, "A Survey of NOMA: Current Status and Open Research Challenges," IEEE Open J. Commun. Soc., vol. 1, pp. 179-189, 2020

[5] T. Assaf, A. J. Al-Dweik, M. S. E. Moursi, H. Zeineldin and M. AlJarrah, "Exact Bit Error-Rate Analysis of Two-User NOMA Using QAM With Arbitrary Modulation Orders," IEEE Commun. Letters, vol. 24, no. 12, pp. 2705-2709, Dec. 2020.

[6] I. Lee and J. Kim, "Average Symbol Error Rate Analysis for NonOrthogonal Multiple Access with M-Ary QAM Signals in Rayleigh Fading Channels," IEEE Commun. Letters, vol. 23, no. 8, pp. 1328-1331, Jun. 2019.

[7] Z. Ding, R. Schober and H. V. Poor, "Unveiling the Importance of SIC in NOMA Systems-Part 1: State of the Art and Recent Findings," IEEE Commun. Letters, vol. 24, no. 11, pp. 2373-2377, Nov. 2020

[8] C-J Kim, Y-S Kim, G-Y Jeong, J-K Mun, H-J Lee, "SER analysis of QAM with space diversity in Rayleigh fading channels," ETRI Journ. vol. 17 , no. 4 , pp. $25-35,1996$

[9] I. L. Shakya, F. H. Ali, and E. Stipidis, "High user capacity collaborative code-division multiple access". IET Commun., vol. 5, no. 3, 307-319, 2011.

[10] W. A. Al-Hussaibi and F. H. Ali, "Efficient User Clustering, Receive Antenna Selection, and Power Allocation Algorithms for Massive MIMONOMA Systems," in IEEE Access, vol. 7, pp. 31865-31882, 2019.

[11] J. G. Proakis, and S. Masoud, Digital Communications, Boston: McGraw-Hill, 2008 